Article

\title{
The Electroluminescence Mechanism of Solution-Processed TADF Emitter 4CzIPN Doped OLEDs Investigated by Transient Measurements
}

\author{
Peng Wang ${ }^{1,2}$, Suling Zhao ${ }^{1,2, *}$, Zheng Xu ${ }^{1,2}$, Bo Qiao ${ }^{1,2}$, Zhijuan Long ${ }^{1,2}$ \\ and Qingyu Huang ${ }^{1,2}$ \\ 1 Institute of Optoelectronic Technology, Beijing Jiaotong University, Beijing 100044, China; \\ 15118435@bjtu.edu.cn (P.W.); zhengxu@bjtu.edu.cn (Z.X.); boqiao@bjtu.edu.cn (B.Q.); \\ 13121561@bjtu.edu.cn (Z.L.); 12118413@bjtu.edu.cn (Q.H.) \\ 2 Key Laboratory of Luminescence and Optical Information, Ministry of Education, \\ Beijing Jiaotong University, Beijing 100044, China \\ * Correspondence: slzhao@bjtu.edu.cn; Tel.: +86-135-1102-3591 \\ Academic Editor: Jwo-Huei Jou \\ Received: 12 September 2016; Accepted: 11 October 2016; Published: 14 October 2016
}

\begin{abstract}
High efficiency, solution-processed, organic light emitting devices (OLEDs), using a thermallyactivated delayed fluorescent (TADF) emitter, 1,2,3,5-tetrakis(carbazol-9-yl)-4,6-dicyanobenzene $(4 \mathrm{CzIPN})$, are fabricated, and the transient electroluminescence (EL) decay of the device with a structure of [ITO/PEDOT: PSS/4CzIPN 5 wt \% doped 4,40-N,N0-dicarbazolylbiphenyl(CBP)/bis-4,6(3,5-di-4-pyridylphenyl)-2-methylpyrimidine (B4PyMPM)/lithium fluoride ( $\mathrm{LiF}$ )/ $\mathrm{Al}$ ], is systematically studied. The results shed light on the dominant operating mechanism in TADF-based OLEDs. Electroluminescence in the host-guest system is mainly produced from the 4CzIPN emitter, rather than the exciplex host materials.
\end{abstract}

Keywords: carrier transport and recombination; transient EL; energy transfer; TADF-based OLEDS

\section{Introduction}

Organic light emitting devices (OLEDs) have been proven successful in the commercial world, including uses in displays and lighting. If the field continues to progress at its current and rapid pace, OLEDs will soon become a mainstay of our technology. However, the future holds even greater promise for this technology, with an entirely new generation of low-cost, lightweight, and flexible electronic devices on offer. External quantum efficiency (EQE) is one of the key parameters for the application of OLEDs [1]. In an OLED, following electron and hole recombination, two types of excitons-singlets and triplets-are generated at a ratio of 1:3, determined by quantum spin-statistics. Fluorescent OLEDs employ spin-antisymmetric singlets for emission, which provides an internal quantum efficiency limit of $25 \%$, and the remaining $75 \%$ of the electrically-generated energy is dissipated via non-radiational methods. As a result, the highest theoretical EQE is restricted to $5 \%$ after considering a light out-coupling constant of $\sim 20 \%$ in devices [2,3].

To achieve OLEDs with very high EQEs, many efforts to utilize triplet excitons have been made in order to break the EQE limit of $25 \%$. It has been demonstrated that electro-phosphorescent organic light-emitting devices (PHOLEDs) can yield a 100\% internal quantum efficiency [4]. Recently, high-efficiency thermally-activated delayed fluorescent (TADF) emitters have been developed as another way to increase the efficiency of OLEDs [3]. TADF OLEDs harvest both singlet and triplet excitons to provide nearly $100 \%$ of the internal conversion of charge into light. In this case, TADF emitters with a small singlet-triplet splitting energy facilitate the thermally-activated reverse intersystem crossing to the singlet manifold, and exerts comparable performance to 
phosphorescent OLEDs [5-8]. Kido et al. developed highly-efficient, solution-processed OLEDs using a TADF emitter1,2,3,5-tetrakis(carbazol-9-yl)-4,6-dicyanobenzene (4CzIPN), as a green emitter [9]. The optimized device showed a very low turn-on voltage of $2.5 \mathrm{~V}$ at $1 \mathrm{~cd} / \mathrm{m}^{2}$, and a relatively high EQE of $16 \%$. They observed the formation of exciplex at the host/electron-transporting layer (ETL) interface, which greatly reduced the operating voltage. This work obtained an outstanding efficiency of solution-processed OLEDs. However, the charge transport and recombination processes in this mixed system are still unintelligent, and the performance roll-off was still remarkable under a high current density. Therefore, it is necessary to fully investigate and understand the fundamental mechanisms in this kind of operating OLED in order to achieve a high performance.

Transient electroluminescence (EL) measurement [10] has been proven to be an effective and profitable method for the study of information on injecting carriers. When OLEDs are driven by the pumping signal of the periodic square wave, instead of being driven by DC bias as usual, the devices provide a delayed luminescence after the end of the forward bias pulse, which is known as delayed EL. Transient EL can be used to analyze the formation of excitons in host-guest systems [11], energy transfer processes [12], and carrier transportation [13] in OLEDs. In this work, we have systematically studied a series of highly-efficient, solution-processed TADF OLEDs with the structure ITO/PEDOT: PSS/4CzIPN doped 4, $4^{\prime}-N, N^{\prime}$-dicarbazolylbiphenyl (CBP, $35 \mathrm{~nm}$ )/ bis-4,6-(3,5-di-4-pyridylphenyl)-2-methylpyrimidine (B4PyMPM, $55 \mathrm{~nm}$ )/lithium fluoride $(\mathrm{LiF}, 6 \AA) / \mathrm{Al}(100 \mathrm{~nm})$, and applied transient electroluminescence measurement in order to investigate the fundamental and physical mechanisms of the operating devices.

\section{Results and Discussion}

We prepared a guest-host system-based device that avoids concentration quenching, similar to conventional fluorescence and phosphorescence. The host in this system plays a highly important role, because the TADF is an up-conversion of triplet excitons into a singlet state-involved process. The emission spectra of OLEDs containing different concentrations of 4CzIPN are shown in Figure 1a. We can see from the EL spectra that the EL peak of CBP-only devices is about $420 \mathrm{~nm}$, which is identified as the exciplex emission, owing to the absence the EL peak of CBP (about $380 \mathrm{~nm}$ ) or B4PyMPM (about $460 \mathrm{~nm}$ ). In the device doped with only $1 \% 4 \mathrm{CzIPN}$, an emission peak is observed at around $500 \mathrm{~nm}$, and is assigned as the emission from the 4CzIPN molecule. The EL emission of 4CzIPN red-shifts along with the increasing concentration, which is due to the interaction between 4CzIPN molecules. It is noted that a small peak at around $420 \mathrm{~nm}$ coinciding with the exciplex emission is observed both in the $1 \mathrm{wt} \%$ and $2 \mathrm{wt} \%$-doped devices. This peak decreased with the increasing concentration of 4CzIPN doping, which means an efficient energy transfer from the interface of the $\mathrm{CBP} / \mathrm{B} 4 \mathrm{PyMPM}$ host to the doped guest. The J-V characteristics of doped and un-doped devices (shown in Figure 1b) are not similar, which suggests that the electroluminescence mechanism is different. As the concentration of $4 \mathrm{CzIPN}$ increases, the current density also increases. This means that $4 \mathrm{CzIPN}$ acts as a transport carrier. According to the energy level of $4 \mathrm{CzIPN}$ (shown in Figure 2), it can transport or trap carriers. If so, the carrier could be recombined directly in $4 \mathrm{CzIPN}$ in order to especially emit in devices with a high 4CzIPN concentration, which is different from the results found in Reference [9].

In order to confirm the electroluminescence mechanism of 4CzIPN, delayed EL measurement was used as an effective method to study the dynamic process of the injecting carriers and excitons in various OLED systems. Measuring the delayed electroluminescence, a persistent EL was found in OLEDs after the electrical bias is turned off, which has been proven to be useful for detecting and identifying various exciton-exciton and carrier-exciton phenomena. In order to enhance the efficient performance and stability of TADF-based OLEDs, it is necessary to gain new insight from the phenomena behind the very high efficiencies attainable in such devices. The time of application of forward and reverse biases is given in Figure 3c; the delayed EL is collected following a $500 \mu$ s forward bias square pulse, where the pulse is sufficiently long to allow the prompt EL 
to reach its steady-state intensity. In Figure 3, a value of 0 on the x-axis corresponds to the time when the forward bias was switched off; this produces the decay luminescence, which is called transient electroluminescence. Generally, the delayed EL in doped OLEDs is raised in the following processes [12-15]: (1) recombination of trapped charges are released at the end of the forward bias pulse; (2) the slow migration of host excitons to neighboring guest sites, where the energy transfer can take place; and (3) the contribution of triplet-triplet-annihilation (TTA). In our prepared devices, the main electroluminescence mechanism is expected as the carrier recombines directly on the TADF material (4CzIPN) for a high efficiency, but not through the energy transfer of exciplex excitons to $4 \mathrm{CzIPN}$. If so, all carriers injected into $4 \mathrm{CzIPN}$ will realize the emission with an efficiency of around $100 \%$. In order to distinguish the definite set of processes occurring in 4CzIPN-based OLEDs, a reverse pulse was applied to prepared devices during the second half of the signal.
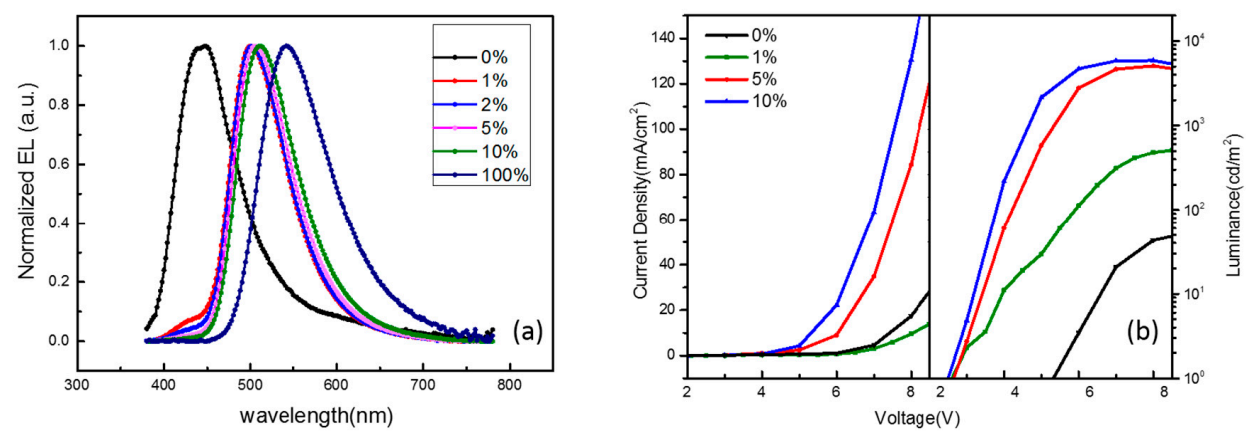

Figure 1. (a) The normalized electroluminescence (EL) spectra of devices with different concentrations of 1,2,3,5-tetrakis(carbazol-9-yl)-4,6-dicyanobenzene (4CzIPN) ( $0 \%$ represents $4,4^{\prime}-N_{,}, N^{\prime}$ dicarbazolylbiphenyl (CBP)-only devices, $100 \%$ represents 4CzIPN-only devices, respectively); (b) current density-voltage-luminance characteristics of the series of organic light-emitting devices (OLEDs).

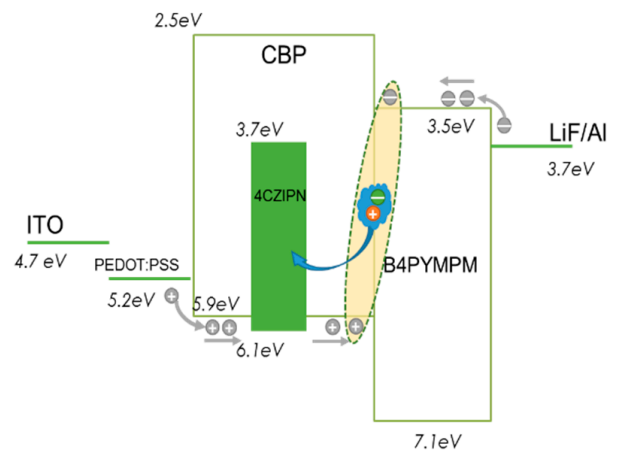

Figure 2. Device structure and energy diagram of organic light emitting devices (OLEDs). B4PyMPM: bis-4,6-(3,5-di-4-pyridylphenyl)-2-methylpyrimidine; ITO: indium tin oxide.
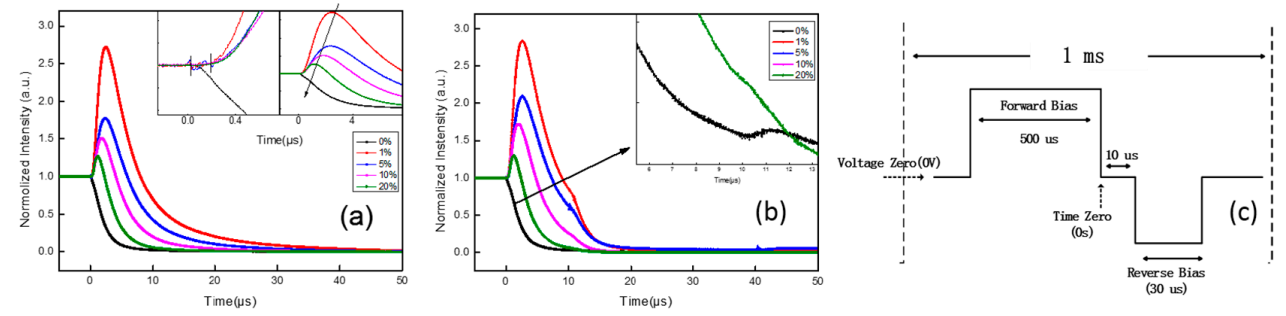

Figure 3. The transient EL curves of $4 \mathrm{CzIPN}$-doped devices with different concentrations; inset is an enlarged view of the transient curves, respectively: (a) without a reverse bias pulse; (b) with a delay time of $10 \mu \mathrm{s}$ and a reverse bias of $5 \mathrm{~V}$; (c) is the timing scheme used in transient EL measurements. 
Figure 3 shows the transient EL curves of $4 \mathrm{CzIPN}$-doped devices with different concentrations. When driven by a periodic square wave bias (Figure 3a), after the bias is switched off, all devices express obvious spikes, except for the OLED with an emitting layer (EML) made of undoped CBP/B4PyMPM. This means that the electroluminescence of $4 \mathrm{CzIPN}$ is not due to the pure energy transfer from the exciplex excitons formed in the interface of CBP/B4PyMPM. Upon closer inspection, with the increase in $4 \mathrm{CzIPN}$ concentrations, the peak intensity gradually decreases, and the time needed to reach its peak is shortened. The transient spikein the EL intensity after the bias turn-off is attributed to the release of trapped charges or residual (i.e., accumulated) charges in the EML.

Under low concentrations, exciton recombination on the host material will play a dominant role. Therefore, the EL luminescence of $4 \mathrm{CzIPN}$ is mainly from the Förster resonance energy transfer (FRET) of the exciplex excitons formed in the host material. The guest molecules act as carrier traps, and the relative trapped charges are released by the sudden change in voltage and lead to the sudden increase of electron-hole recombination to yield an increase in EL intensity. As the 4CzIPN concentration increases, the exciton formation on the host decreases, the energy transfer from the exciplex is reduced, and the direct recombination of carriers injected into $4 \mathrm{CzIPN}$ hold the dominant position. This implies the reduction of carrier traps; therefore, the EL intensity spike is weakened with the increase in $4 \mathrm{CzIPN}$ concentration. In addition, the guest molecule concentration increases the probability that the recombination of trapped carriers was increased, leading to the earlier attainment of peak intensity.

As shown in Figure 3b, a reverse pulse with a width of $30 \mu$ s was applied to the devices with a delay time of $10 \mu$ s behind the forward bias, which is longer than the typical lifetime of singlet excited-state 4CzIPN ( 20 ns). The doped devices showed an accelerated EL decay under the reverse bias, compared with Figure 3a, while the undoped device experienced a partial increase when the reverse pulse was on. The joining of the reverse bias sped up the dissociation of the exciton in $4 \mathrm{CzIPN}$ formed under a forward bias. If the transient EL is induced by the direct recombination of injected charges, a sudden decrease will appear when the reverse bias is applied; however, there will be no recovery at the ending edge of the reverse pulse compared with TTA [13]. If the exciton of $4 \mathrm{CzIPN}$ is formed by the energy transfer from the interfacial exciplex exciton, the transient EL intensity form of the devices doped with $4 \mathrm{CzIPN}$ should depend on that of the device without $4 \mathrm{CzIPN}$. The difference in the EL decay from doped and undoped devices means that the energy transfer between the interfacial exciplex to $4 \mathrm{CzIPN}$ is not the main process for the emission of $4 \mathrm{CzIPN}$, especially in highly-doped devices. Therefore, it is concluded that the main mechanism of the electroluminescence of the $4 \mathrm{CzIPN}$-doped CBP/B4PyMPM system is the direct recombination of the injected carrier of 4 CzIPN.

For further investigation, reverse biases with different magnitudes were applied to these devices, and results are shown in Figure 4. As can be seen in Figure 4a, increasing the magnitude of the reverse bias results in an increase of the spike at the beginning of the reverse bias in the device without $4 \mathrm{CzIPN}$. This suggests that, in exciplex emitting devices, the delayed emission is primarily due to the recombination of released trap charges [12] at the interface between CBP and B4PyMPM, under the applied reverse voltage. In contrast, the increase in reverse bias speeds up the dissociation of excitons from 4 CzIPN, causing a decrease in the decay curves in doped devices (shown in Figure $4 b-d$, respectively. The transient EL of $20 \mathrm{wt} \%$ doped devices are not shown here, as the data are similar to those doped at $10 \mathrm{wt} \%$ ). Based on the systematic investigation of delayed EL, the main mechanism of $4 \mathrm{CzIPN}$ emission is due to the carrier recombination of $4 \mathrm{CzIPN}$. The energy transfer process in these doped devices is neglected, especially when the concentration of $4 \mathrm{CzIPN}$ is high. 

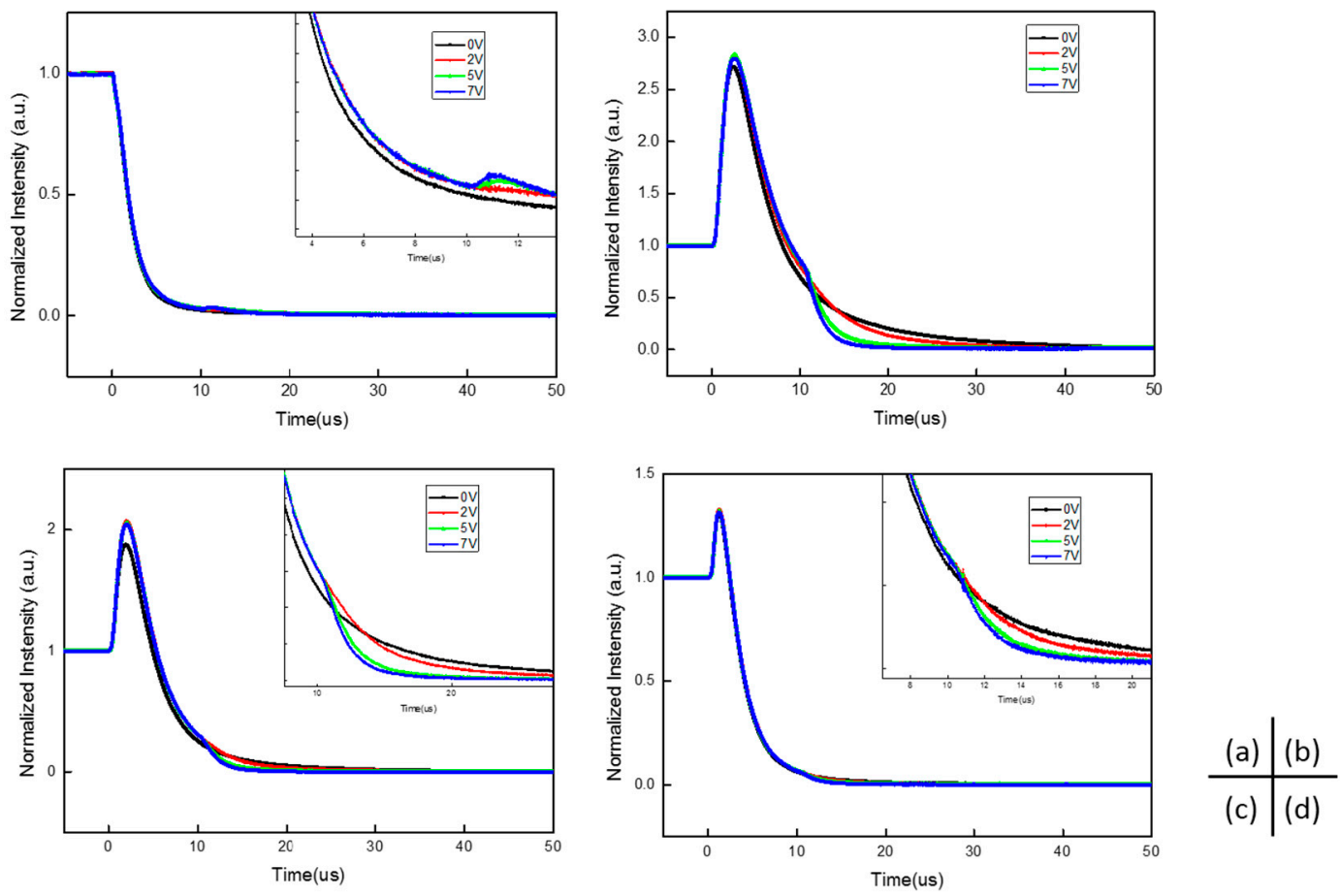

Figure 4. The transient EL curves of $4 \mathrm{CzIPN}$ doped devices with different reverse bias; inset is the enlarged view. (a) Is for the un-doped devices; (b-d) are 1\%, 5\%, and 10\% doped devices, respectively.

\section{Experimental Section}

The structure of the TADF devices and the principal materials used in this work are shown in Figure 2. CBP, which exhibits bipolar transmission capability, was used as the host for 4CzIPN, a typical TADF molecule [3]. A wide energy gap electron transporter, B4PyMPM, was used as the ETL. The emitting layer (EML) was spin-coated from a chlorobenzene solution $(10 \mathrm{mg} / \mathrm{mL})$ in a nitrogen atmosphere; the ETL and cathode were fabricated using thermal evaporation at a deposition rate of $1-2 \AA / s$ in vacuum, with a pressure of about $2 \times 10^{-4} \mathrm{~Pa}$. Doping concentrations of $4 \mathrm{CzIPN}$ in CBP are $1,5,10$, and $20 \mathrm{wt} \%$, respectively. The transient electroluminescence properties were detected using a Zolix Instruments Model PMTH-S1C1-CR131 Photomultiplier Tube. The time evolution of delayed light emission was recorded with a Tektronix Model DPO 4104 digital phosphor oscilloscope. All measurements were performed at room temperature under an atmospheric environment.

\section{Conclusions}

We fabricated solution-processed OLEDs using a TADF emitter 4CzIPN-doped CBP/B4PyMPM system. It is worth noting that the formation of exciplex at the host/ETL interface can greatly reduce the operating voltage, and thus significantly improves device performance. We also studied the corresponding transient EL decay of this system. The results provide direct evidence to identify the dominant operating mechanism in TADF-based OLEDs. The main mechanism of the electroluminescence of the 4CzIPN-doped CBP/B4PyMPM system is the injected carrier recombination of $4 \mathrm{CzIPN}$. The energy-transfer process in these doped devices is neglected, especially when the concentration of $4 \mathrm{CzIPN}$ is high.

Acknowledgments: We greatly acknowledge partial financial support from the 863 Program (2013AA032205), the NSFC (51272022 and 11474018), RFDP (20120009130005), and FRFCU (2012JBZ001).

Author Contributions: S.Z. and P.W. conceived and designed the experiments; P.W. performed the experiments; Z.L. and Q.H. analyzed the data; Z.X. and B.Q. contributed reagents/materials/analysis tools; P.W. wrote the paper.

Conflicts of Interest: The authors declare no conflict of interest. 


\section{References}

1. Adachi, C. Third-generation organic electroluminescence materials. Jpn. J. Appl. Phys. 2014. [CrossRef]

2. Endo, A.; Ogasawara, M.; Takahashi, A.; Yokoyama, D.; Kato, Y.; Adachi, C. Thermally activated delayed fluorescence from $\mathrm{Sn}(4+)$-porphyrin complexes and their application to organic light emitting diodes-A novel mechanism for electroluminescence. Adv. Mater. 2009. [CrossRef] [PubMed]

3. Uoyama, H.; Goushi, K.; Shizu, K.; Nomura, H.; Adachi, C. Highly efficient organic light-emitting diodes from delayed fluorescence. Nature 2012. [CrossRef] [PubMed]

4. Baldo, M.A.; O’brien, D.F.; You, Y.; Shoustikov, A.; Sibley, S.; Thompson, M.E.; Forrest, S.R. Highly efficient phosphorescent emission from organic electroluminescent devices. Nature 1998, 395, 151-154.

5. Goushi, K.; Yoshida, K.; Sato, K.; Adachi, C. Organic light-emitting diodes employing efficient reverse intersystem crossing for triplet-to-singlet state conversion. Nat. Photonics 2012. [CrossRef]

6. Lin, T.-A.; Chatterjee, T.; Tsai, W.-L.; Lee, W.-K.; Wu, M.-J.; Jiao, M.; Pan, K.-C.; Yi, C.-L.; Chung, C.-L.; Wong, K.-T.; et al. Sky-Blue Organic Light Emitting Diode with 37\% External Quantum Efficiency Using Thermally Activated Delayed Fluorescence from Spiroacridine-Triazine Hybrid. Adv. Mater. 2016. [CrossRef] [PubMed]

7. Shiu, Y.-J.; Cheng, Y.-C.; Tsai, W.-L.; Wu, C.-C.; Chao, C.-T.; Lu, C.-W.; Chi, Y.; Chen, Y.-T.; Liu, S.-H.; Chou, P.-T. Pyridyl Pyrrolide Boron Complexes: The Facile Generation of Thermally Activated Delayed Fluorescence and Preparation of Organic Light-Emitting Diodes. Angew. Chem. Int. Ed. 2016. [CrossRef] [PubMed]

8. Tsai, W.-L.; Huang, M.-H.; Lee, W.-K.; Hsu, Y.-J.; Pan, K.-C.; Huang, Y.-H.; Ting, H.-C.; Sarma, M.; Ho, Y.-Y.; $\mathrm{Hu}, \mathrm{H}$.-C.; et al. A versatile thermally activated delayed fluorescence emitter for both highly efficient doped and non-doped organic light emitting devices. Chem. Commun. 2015. [CrossRef] [PubMed]

9. Komatsu, R.; Sasabe, H.; Inomata, S.; Pu, Y.-J.; Kido, J. High efficiency solution processed OLEDs using a thermally activated delayed fluorescence emitter. Synth. Met. 2015. [CrossRef]

10. Long, Z.; Zhao, S.; Xu, Z.; Wang, P.; Zhang, C.; Sun, L. The electroluminescence mechanism of non-doping PhOLEDs based on CBP/Ir(ppy)3 investigated by delayed EL measurements. Org. Electron. 2016. [CrossRef]

11. Wang, S.; Zhang, Y.; Chen, W.; Wei, J.; Liu, Y.; Wang, Y. Achieving high power efficiency and low roll-off OLEDs based on energy transfer from thermally activated delayed excitons to fluorescent dopants. Chem. Commun. 2015. [CrossRef] [PubMed]

12. Song, D.; Wang, Q.; Zhao, S.; Aziz, H. Dependence of carrier recombination mechanism on the thickness of the emission layer in green phosphorescent organic light emitting devices. Org. Electron. 2011. [CrossRef]

13. Huang, Q.; Zhao, S.; Xu, Z.; Fan, X.; Shen, C.; Yang, Q. Exciplex emission and decay of co-deposited 4, $4^{\prime}, 4^{\prime \prime}$-tris3-methylphenyl(phenyl)aminotriphenylamine:tris-3-(3-pyridyl)mesitylborane organic light-emitting devices with different electron transporting layer thicknesses. Appl. Phys. Lett. 2014. [CrossRef]

14. Song, D.; Zhao, S.; Luo, Y.; Aziz, H. Causes of efficiency roll-off in phosphorescent organic light emitting devices: Triplet-triplet annihilation versus triplet-polaron quenching. Appl. Phys. Lett. 2010. [CrossRef]

15. Zhang, C.; Zhao, S.; Xu, Z.; Hong, X.; Long, Z.; Wang, P.; Chen, Y.; Xu, X. The storage of charges and its optical application in organic light-emitting diodes measured by a transient electroluminescence method. Org. Electron. 2015. [CrossRef]

Sample Availability: Samples of the compounds are not available from the authors. 